\title{
Effects of music intervention during caesarean delivery on anxiety and stress of the mother a controlled, randomised study
}

\author{
Philip Hepp ${ }^{1,2^{*}}$ (D), Carsten Hagenbeck ${ }^{2}$, Julius Gilles ${ }^{2}$, Oliver T. Wolf ${ }^{3}$, Wolfram Goertz ${ }^{4}$, Wolfgang Janni ${ }^{5}$,
}

Percy Balan², Markus Fleisch', Tanja Fehm ${ }^{2}$ and Nora K. Schaal ${ }^{6}$

\begin{abstract}
Background: Stress and anxiety during pregnancy and childbirth have negative consequences for both mother and child. There are indications that music has a positive effect in this situation. The present study investigates the influence of music during the caesarean on anxiety and stress of the expectant mother.

Methods: The SAMBA study is a single-centre, controlled, randomized study including 304 patients. Women in the intervention group heard music via loudspeakers from one of four self-selected genres. The control group had standard treatment without music. The caesarean was performed in regional Anesthesia. At admission, at skin incision, during skin suture and two hours after completion of surgery, different subjective (State-Trait Anxiety Inventory, visual analogue scale for anxiety) and objective parameters (salivary cortisol/amylase, heart rate, blood pressure) were collected. Mixed-factorial Analysis of variances as well as independent sample t-tests were applied for data analysis.
\end{abstract}

Results: At skin suture, significantly lower anxiety levels were reported in the intervention group regarding State anxiety (31.56 vs. $34.41 ; p=.004)$ and visual analogue scale for anxiety (1.27 vs. 1.76; $p=.018)$. Two hours after surgery, the measured visual analogue scale for anxiety score in the intervention group was still significantly lower (0.69 vs. 1.04; $p=.018$ ). The objective parameters showed significant differences between the groups in salivary cortisol increase from admission to skin suture (12.29 vs. $16.61 \mathrm{nmol} / \mathrm{L} ; p=.043)$, as well as systolic blood pressure (130.11 vs. $136.19 \mathrm{mmHg} p=.002)$ and heart rate $(88.40$ vs. $92.57 / \mathrm{min} ; p=.049)$ at skin incision.

Conclusions: Music during caesarean is an easy implementable and effective way of reducing stress and anxiety of the expectant mother.

Trial registration: German registry for clinical trials (DRKS00007840). Registered 16/06/2015. Retrospectively registered. Keywords: Caesarean, Anxiety, Stress, Music intervention

\section{Background}

Almost one in three women delivers by caesarean in Germany. Thus it is the most common abdominal surgery and one of the most common operations. Although the circumstances in almost all cases give cause for joy, it is also a feared event, which is associated with a

\footnotetext{
* Correspondence: science@dr-hepp.de

${ }^{1}$ Clinic for Gynecology and Obstetrics, Helios University Hospital Wuppertal, University Witten/Herdecke, Heusnerstr 40, 42283 Wuppertal, Germany

${ }^{2}$ Clinic for Gynecology and Obstetrics, Heinrich-Heine-University, Düsseldorf, Germany

Full list of author information is available at the end of the article
}

significant level of stress for the patient $[1,2]$. Studies have shown adverse effects of maternal stress on the foetus and on psychological development later in life [3, 4]. In addition, it is known that increased levels of stress and anxiety can negatively affect pain perception and the usage of analgesics postoperatively $[5,6]$ as well as the new mothers lactation $[7,8]$. In view of the limited pharmacological options of intervention for pregnant women, the need for alternative, low-risk approaches to positively influence anxiety and stress arises.

In this regard, the positive effect of music on anxiety and stress is one of the oldest treatment approaches. 
Even Asclepius attributed a healing power to music [9]. Its positive influence in various medical interventions across all disciplines has been repeatedly examined [10-13]. Supported by a recent review by Hole et al. in the Lancet, these results also drew the attention of the wider public [14].

Nevertheless, data for music during caesarean are sparse and inconclusive [15]. Two randomized studies examining the influence of music during the pre-operative waiting time could show a positive influence of music on non-validated questionnaires as well as heart rate and heart rate variability as a surrogate for stress and anxiety. In part, this effect could still be detected six hours after the caesarean $[16,17]$. A Cochrane analysis [15] investigating the effect of music during the procedure could only identify one randomized study that was able to show a positive effect of music intervention on heart rate at the end of the caesarean in 64 participants [18]. Due to their study design, no further conclusions on subjective and objective perception of anxiety were possible. Another study examined the influence of music during caesarean delivery under general anesthesia on postoperative pain and could not show any effect [19].

Therefore, the aim of the present study is to systematically examine the anxiolytic and stress reducing effect of a music intervention during the caesarean on the wake patient using validated questionnaires and a comprehensive set of objective measurements (salivary cortisol/amylase, blood pressure and heart rate).

\section{Methods}

The $S A M B A$ (Sectio Caesarea und die Auswirkung von MusikBegleittherapie auf Anxiolyse; English: Caesarean and the effect of music intervention on anxiety) study is a single-centre, controlled, randomized trial conducted at the University Hospital Dusseldorf, Germany. The study adheres to the CONSORT guidelines.

\section{Ethics, consent and permissions}

The study protocol was approved by the ethics committee of the Medical Department of the Heinrich-Heine-University in Dusseldorf (No.: 3625) in accordance with the declaration of Helsinki and registered in the German registry for clinical trials (DRKS00007840) and with the WHO (Universal Trial Number U1111-1173-3204). Upon reasonable request the study protocol can be obtained via email from the corresponding author. All eligible patients gave informed written consent prior to participation.

\section{Participants}

From March 2015 to August 2017, pregnant women with an indication for primary caesarean in regional anesthesia and adequate German language comprehension were recruited.
We only included patients with normal hearing abilities. Additionally, the patients were only included when no serious comorbidities (according to the physician's assessment) were present, no significantly increased surgical risk (e.g. placental disturbances) were identified preoperatively and only if no serious condition of the foetus was known. Furthermore, only patients without any generalized anxiety disorder or other serious mental alterations were included in the study.

\section{Outcomes, measuring instruments and procedure}

The impact of music during caesarean delivery on anxiety and stress measured by State-Trait Anxiety Inventory (STAI), a visual analogue scale depicting anxiety (VAS-A) as well as on salivary cortisol and alpha-amylase were pre-specified as primary outcome measures.

To measure subjective anxiety, STAI and VAS-A were used. The STAI is an introspective inventory comprising 40 self-report items pertaining to anxiety [20]. It distinguishes between two questionnaires with 20 items each, one measuring anxiety perceived in the current situation (STAI-state) and the other evaluating a general tendency towards anxiety (STAI-trait). Participants are asked to give a response to each item on a 4-point Likert scale. A total value is calculated (possible range 20-80 for each questionnaire). Higher scores reflect higher levels of anxiety. The VAS-A comprises a $10 \mathrm{~cm}$ line, on which the participant marks her current degree of anxiety with the left end of the line being labelled "no anxiety" and the right end being labelled "maximum anxiety". For analysis the marking is then measured in $\mathrm{mm}$ from the left end.

Saliva samples were collected in order to determine cortisol and alpha-amylase levels as objective measures of stress. Salivary cortisol is a marker of the activation of the hypothalamic-pituitary-adrenal axis, whereas salivary alpha-amylase is an indirect marker of the autonomic activity [21]. For the saliva samples, patients had to thoroughly insalivate a cotton swab. The saliva samples were kept frozen at - 18 degrees until analysed, following the methods described elsewhere [22]. Heart rate and blood pressure values were taken from the anaesthesia records.

After inclusion, the computer-assisted randomization took place and divided the patients into the music group vs. control group in the ratio of one to one. Women in the music group chose their preferred music genre from lounge, classical, jazz, and meditation music.

The participating women filled in the STAI-trait when they came to the routine surgical preparation appointment 7 to14 days before the caesarean. At admission on the day of the scheduled caesarean, the VAS-A, the STAI-state and the first saliva sample were taken during the routine cardiotocogram. There was no routine preoperative medication in either group. Intraoperative, the 
parameters blood pressure and heart rate were recorded at skin incisions and suture. During skin suture, a second saliva sample was obtained and the patient answered the STAI-state questionnaire and the VAS-A. At the end of post-operative monitoring two hours after skin suture, a third saliva sample was taken. Furthermore, the participant answered the STAI-state questionnaire and VAS-A for a third time. Additionally, questions were asked about the music experience. Figure 1 shows the study procedure with the measurement time points schematically.

\section{Intervention}

For the music group, the music intervention started when the participant entered the operating theatre. The music was played on a CD player (TEAC CR-H $500 \mathrm{CD}$ receiver) using a speaker system from Cambridge Audio 300. The participant was presented with music continuously in a standardized volume determined on the device and measured at the participants' head of $55 \mathrm{~dB}$ (A). All music titles had a slow tempo of $60-80 \mathrm{bpm}$ in common and followed the recommendations made by Nilsson [23]. The music pool contained 60 songs. 15 tracks were each assigned to one of the 4 different genres of music. The control group received no music.

\section{Statistical analysis}

The statistical software package SPSS 24 (IBM Inc., Armonk, NY) was used for all data analyses. The group affiliation was coded and therefore the analysis was performed blind. In order to check for differences in environmental parameters between groups, which could influence the results, we compared the time of the procedure (morning vs. afternoon) using a chi-square-test and the length of the surgery with an independent sample t-test.

To compare the subjective course of anxiety on the day of the caesarean, two $2 \times 3$ mixed-factorial ANOVAs with the between-subject factor group (music group vs control group) and the within-subject factor measurement time point (admission, skin suture, $2 \mathrm{~h}$ post-surgery) and the dependent variables STAI-state and VAS-A were calculated. If sphericity was not met, a correction of the degrees of freedom according to Greenhouse-Geisser was carried out. For the objective variables heart rate and systolic and diastolic blood pressure, $2 \times 2$ mixed-factorial ANOVAs were used with the factors group and measurements time point (skin incision and skin suture). In addition, direct group comparisons were performed with independentsamples t-tests. Amylase values were logarithmized analogously to the generally accepted approach [24].

An a-priori power analyses for sample-size estimation was calculated using G*Power (HHU, Düsseldorf, Germany) [25]. Given an expected small to medium effect size $(\mathrm{d}=$ 0.35 ), a power of $85 \%$ and an alpha-error of .05 the required (to be analysed) sample-size is 296 (148 per group).

\section{Results}

We screened and informed 412 patients about study participation. Sixty-two patients had to be excluded from further participation because they no longer fulfilled the inclusion criteria at the time of intervention (three had delivered spontaneously, 18 had an indication for caesarean preterm, 41 had a secondary caesarean (for example because of premature rupture of membranes)). Forty-five women did not take part due to technical difficulties (reconstruction of the operation theatre). One patient discontinued the study prematurely. In total 304 participants completed the study in accordance with the protocol (Fig. 2). The patients had a mean age of 33.6 years (range: 18-47 years) and a mean gestation age of 268.5 days. The data revealed that the two groups did not differ regarding the time of the procedure (morning vs. afternoon) $\left[\chi^{2}(1\right.$, $301)=0.75, p=.388]$ nor on the duration of the procedure $[t(290)=1.36, p=.175]$.

An overview of the descriptive data of the music and control group and the main results is given in Table 1.

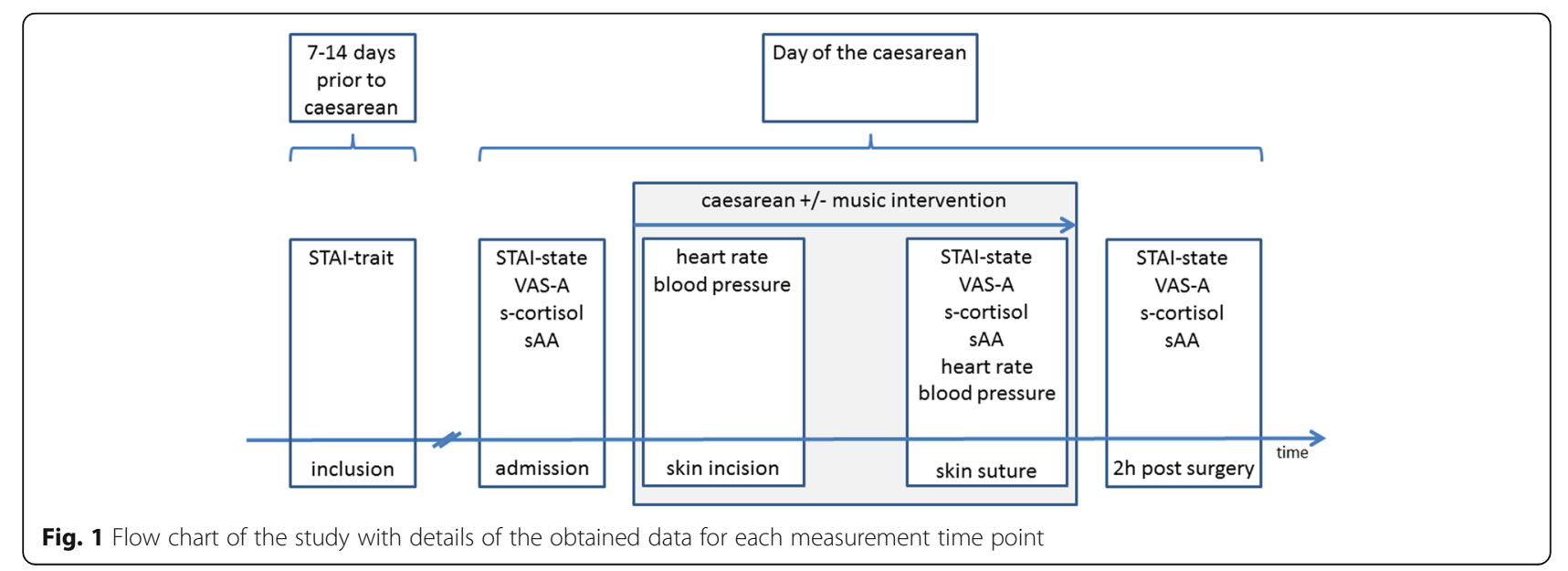




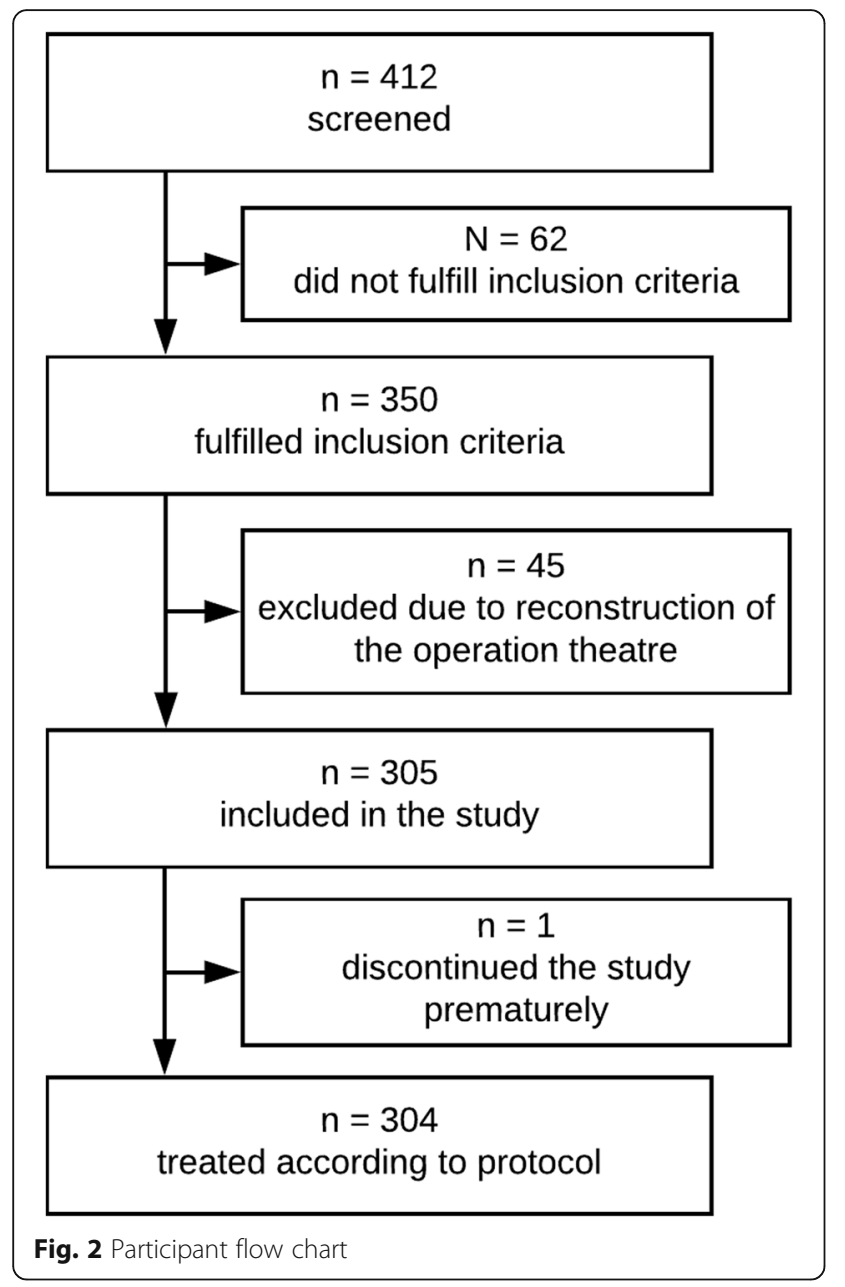

\section{Subjective parameters}

For the STAI-state, significant main effects were found for the factors measurement time point $[F(1.78,451.28)=$ $454.35, p<.001]$ and group $[F(1.254)=4.12, p=.043]$. The interaction was not significant $[F(1.78,451.28)=$ 1.47, $p=.230$ ]. Post-hoc comparisons showed that the two groups did not differ on admission $(p=.593)$. At skin suture the music group showed significantly less anxiety compared to the control group $[t(254)=2.88, p=.004$, mean difference $=2.72,95 \% \mathrm{CI}(0.76,4.67), \mathrm{d}=0.36] .2 \mathrm{~h}$ after surgery there was no significant difference $(p=.140)$ (Fig. 3a).

For the VAS-A, the factors measurement time point [ $F$ $(1.39,301.32)=378.50, p<.001]$ and group $[F(1.217)=$ $4.51, p=.035]$ showed significant effects, whereas no interaction was present $[F(1.39,301.32)=0.17, p=.847]$. A post-hoc t-test showed that the level of anxiety in the two groups did not differ at admission $(p=.349)$. At skin suture $[t(217)=2.39, p=.018$, mean difference $=0.49,95 \% \mathrm{CI}$ $(0.08,0.89), \mathrm{d}=0.32]$ and $2 \mathrm{~h}$ post-surgery $[t(217)=2.38$, $p=.018$, mean difference $=0.35,95 \%$ CI $(0.06,0.64)$, $\mathrm{d}=0.32]$ there were significant differences in favour of the music group (Fig. 3b).

\section{Objective parameters}

Cortisol increase from admission to skin suture was significantly lower in the music group than in the control group $[t(181)=2.04, p=.043$, mean difference $=4.32$, 95\% CI (0.14, 8.49), $d=0.30$ ] (Fig. 4a). The decrease in cortisol from skin suture to $2 \mathrm{~h}$ post-surgery did not differ significantly between the two groups [ $\mathrm{t}(193)=1.53$, $p=.121$, mean difference $=3.30,95 \%$ CI $(-0.95,7.55)$, $\mathrm{d}=0.22]$. The increase in amylase from admission to skin suture and decrease to $2 \mathrm{~h}$ after surgery did not differ between the two groups ( $p$-values $>.165$ ).

For systolic blood pressure, significant main effects were found for group $[F(1,270)=4.73, p=.030]$ and measurement time point $[F(1,270)=132.58, p<.001]$ and a significant interaction revealed $[F(1,270)=8.57, p=.004]$. At skin incision the mean systolic blood pressure was significantly lower in the music group $(\mathrm{M}=130.11 \mathrm{mmHg})$ than in the control group $(\mathrm{M}=136.19 \mathrm{mmHg})[t(270)=3.18$, $p=.002$, mean difference $=6.35,95 \%$ CI $(2.59,10.11), \mathrm{d}=$ 0.39] (Fig. 4b). There was no difference at skin suture $(p=.981)$. For the diastolic blood pressure value, no significant group differences were found ( $p$-values $>.197$ ).

Also for heart rate significant effects of the factors group $[F(1,266)=4.51, p=.035]$ and measurement time point $[F(1,266)=104.99, p<.001]$ were revealed. There was no significant interaction $[F(1,266)=0.55, p=.457]$. Post-hoc comparisons showed that at skin incision the heart rate was significantly lower in the music group $(\mathrm{M}=88.40)$ than in the control group $(\mathrm{M}=92.57)$ [ $\mathrm{t}$ $(269)=1.98 p=.049$, mean difference $=4.13,95 \% \mathrm{CI}$ $(0.02,8.24), d=0.24]$. At skin suture, the groups did not differ significantly $(p=.141)$ (Fig. 4c).

\section{Acceptance of music intervention}

Of the participants in the music group $95.5 \%$ stated that they would listen to music again at a possible next caesarean. Eighty-six percent were satisfied with the choice of music. In addition, $89.7 \%$ said that the music made the situation more enjoyable and $73.4 \%$ thought the music had calmed them.

\section{Discussion}

Overall, the study shows, in subjective as well as objective dimensions, an anxiety and stress reducing effect of music during caesarean.

While both groups showed high baseline values for STAI-state and VAS-A without significantly differing from each other at admission, the subjective anxiety drop in both, STAI-state and VAS-A, was significantly higher in the music group and resulted in significantly lower anxiety scores compared to the control group at 
Table 1 Overview of characteristics and results

\begin{tabular}{|c|c|c|c|}
\hline & Music group & Control group & $P$ value \\
\hline Number & 154 & 150 & \\
\hline Age [years] & $33.5 \pm 5.4(18-47)$ & $33.7 \pm 5.4(21-44)$ & .883 \\
\hline Gestational Age [days] & 269 & 268 & .231 \\
\hline Time of procedure [morning/evening] & $81 / 73$ & 70/77 (3 N/A) & .388 \\
\hline Duration of procedure [mins] & 43.0 & 41.6 & .175 \\
\hline STAI-Trait & $36.39 \pm 8.45$ & $37.14 \pm 8.74$ & .474 \\
\hline STAI-State I & $47.54 \pm 10.40$ & $48.28 \pm 11.79$ & .593 \\
\hline STAI-State II & $31.56 \pm 6.30$ & $34.41 \pm 9.23$ & .004 \\
\hline STAI-State III & $29.54 \pm 5.91$ & $30.91 \pm 7.14$ & .140 \\
\hline VAS-A I [cm] & $4.83 \pm 2.61$ & $5.18 \pm 2.89$ & .349 \\
\hline VAS-A $\|[\mathrm{cm}]$ & $1.27 \pm 1.20$ & $1.76 \pm 1.78$ & .018 \\
\hline VAS-A III [cm] & $0.69 \pm 0.88$ & $1.04 \pm 1.29$ & .018 \\
\hline C-increase | to || [nmol/L] & $12.29 \pm 12.15$ & $16.61 \pm 16.14$ & .043 \\
\hline C-decrease II to III [nmol/L] & $-13.77 \pm 13.05$ & $-17.07 \pm 16.80$ & .128 \\
\hline sAA-increase $[\mathrm{In}]$ | to || & $1.58 \pm 1.40$ & $1.76 \pm 1.24$ & .414 \\
\hline sAA-decrease II to III & $-0.31 \pm 1.27$ & $-0.57 \pm 1,10$ & .165 \\
\hline RR sys skin incision [mmHg] & $130.11 \pm 14.97$ & $136.19 \pm 16.57$ & .002 \\
\hline RR sys II [mmHg] & $121.42 \pm 12.89$ & $121.58 \pm 13.00$ & .981 \\
\hline RR dia skin incision [mmHg] & $70.82 \pm 9.35$ & $72.77 \pm 10.55$ & .106 \\
\hline RR dia II [mmHg] & $64.65 \pm 9.61$ & $64.99 \pm 9.21$ & .764 \\
\hline HR skin incision [1/min] & $88.40 \pm 16.23$ & $92.57 \pm 18.26$ & .049 \\
\hline $\mathrm{HR}$ II [1/min] & $77.97 \pm 13.91$ & $80.51 \pm 14.23$ & .141 \\
\hline
\end{tabular}

N/A: data not available; I: at admission; II: at skin suture; III: $2 \mathrm{~h}$ post-surgery; VAS-A: Visual Analogue Scale for Anxiety; C: salivary cortisol; sAA: salivary alpha amylase; RR sys: systolic blood pressure; RR dia: diastolic blood pressure; HR: heart rate Note: bold values indicate a significant difference between groups

skin suture. The shown positive effect of music on these subjective measures for anxiety and stress is in line with previous studies investigating the effect of music in the context of caesarean and other medical procedures $[11,13,14,16,23,26]$. One of these studies showed a persisting positive effect for six hours [16]. Our data support this observation insofar that women in the music group had significantly lower scores on the VAS-A for another two hours after skin suture. This effect was not found for the STAI-state. It is likely that the STAI-state is less appropriate than the VAS-A in the obstetrical environment due to the duration of the survey?

In terms of objective parameters, the music group showed a significantly lower increase in salivary cortisol
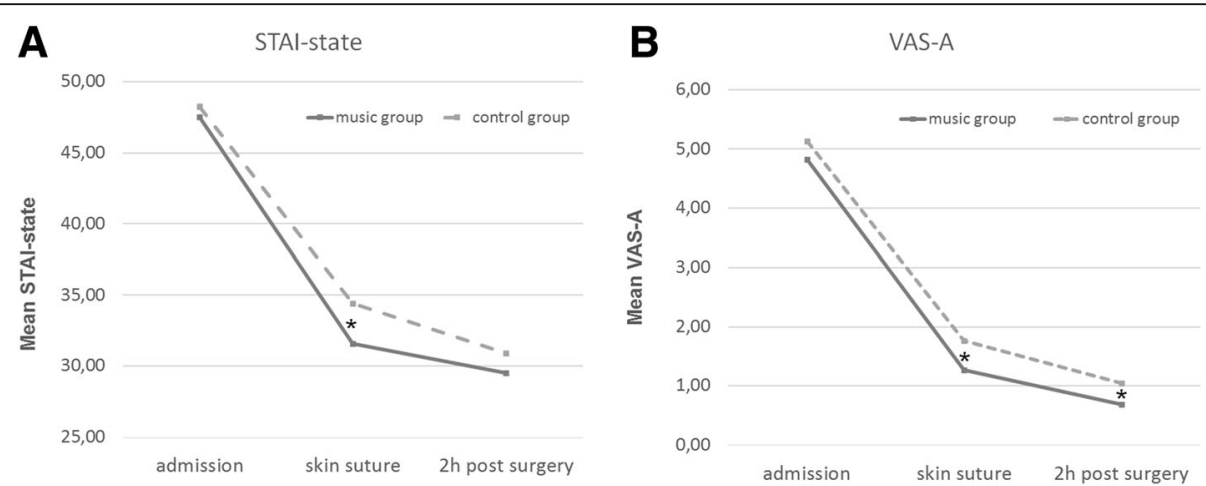

Fig. 3 The subjective course of anxiety for both groups. a For the STAl-state scores the music group displayed significantly lower anxiety levels at skin suture than the control group. $\mathbf{b}$ For the VAS-A scores anxiety levels of the music group are significantly below the control group at skin suture and remain lower two hours after the caesarean. ${ }^{*} p<.05$ 

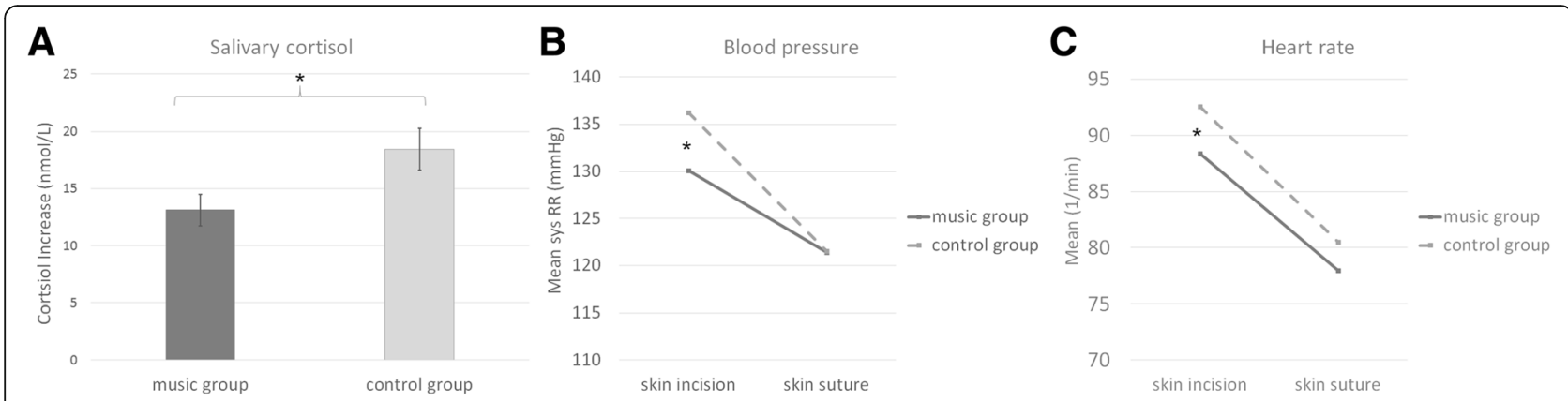

Fig. 4 Objective Measures. a The increase of salivary cortisol from admission to skin suture is significantly higher in the control group than the music group. $\mathbf{b}$ The music group show significantly lower systolic blood pressure at skin incision. $\mathbf{c}$ Heart rate is significantly lower in the music group than the control group at skin incision

than the control group from admission to skin suture. Saliva cortisol measures the impact of a stressor with a latency of about $30 \mathrm{~min}$ [27]. In this respect, the measurement at skin suture represents a large part of the objective stress sensation during the operation [2]. Therefore, the present result reflects that the music group also objectively experiences reduced stress levels during the caesarean. A lowering effect of music on cortisol could also be shown in studies in other medical fields [28].

A comparable effect could not be found for salivary amylase. Whereas cortisol represents the activity of the hypothalamic-pituitary-adrenocortical axis, salivary amylase represents the activation of the sympathetic nerve system [29]. In line with previous work on stress during surgical procedures, our study shows no measurable difference in salivary amylase between the groups $[30,31]$. This might be an indication that there are unrecognized factors, which impede a reliable interpretation of the salivary amylase during surgical procedures.

A variety of studies have examined the influence of music on the cardiovascular system [32]. In addition to a direct impact on the dopaminergic mesolimbic reward centre [33], an interaction of the external musical rhythm with the internal body rhythms of heart and respiratory rate as a main carrier of the effect of music is debated [34-36]. Thereby, the value of systolic blood pressure and heart rate is well documented as objective parameters reflecting stress and anxiety [32]. In the present study, the music group showed significantly lower heart rate and systolic blood pressure levels at skin incision. This took place on average $18 \mathrm{~min}$ after the patient had been admitted to the operating room and the start of the music intervention. Therefore, we would argue that the calming effect of music on the expectant mother was already present at skin incision. In the case of skin suture, however, no significant difference was detectable. At this time, the new-born was usually already in the arms of the mother, so that pulse and blood pressure are certainly subject to an undetermined bias.
Besides the positive influence of music on the measured stress and anxiety of patients, the high acceptance of the intervention should also be noted. Ninety-six percent of women in the intervention group would want to hear music again during a possible repeated caesarean, regardless of any therapeutic effects. The positive response of the women regarding the music intervention alone are gratifying and should encourage the use of music in obstetrics and further research in this area.

A limitation of the study is the lack of opportunity to gain an even more comprehensive picture of the course of anxiety during caesarean through measuring more time points. For example a measurement of anxiety levels just before the women enters the operating theatre would be desirable as this time point may be the most anxious time for the women. This is partially compensated by the fact that the different measuring instruments used in this study have different latencies and thus cover a large period of time around the intervention. Additionally, in order to ensure a trial close to normal conditions of a caesarean and the needs of the woman, we decided not to use headphones, in contrast to other studies [18]. Naturally, this impeded blinding of the study staff. Using group blinded analysis and application of objective parameters such as saliva cortisol, this disadvantage was accounted for as far as possible. Another limitation which could have influenced the study results is the lack of data on the relationship and role of the nurses and other health professionals in usual interaction and information sharing with the woman during and after procedure.

We would like to emphasize that with a large sample of 304 participants and several measurement time points throughout the day of the caesarean as a strength of the study. This is the first study in this area of research that has the necessary power to detect even small effect sizes.

\section{Conclusions}

In view of the results, the present study should have implications for clinical practice. Music is a simple, 
inexpensive, effective and safe intervention that can be easily implemented in everyday clinical practice. Therefore, it would be desirable if the possibility to listen to music could be routinely offered to women giving birth by caesarean. However, further research should consider the impact of music on the surgical team. Although previous work showed adverse effects only for high volumes and complex interventions [37], this has not been investigated explicitly in the context of caesarean.

In conclusion, the results of the present study show that music during caesarean has an anxiety and stress soothing effect on the wake patient. Implementation in clinical routine therefore seems advisable.

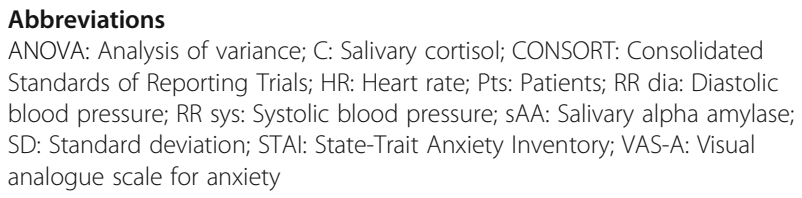

ANOVA: Analysis of variance; C: Salivary cortisol; CONSORT: Consolidated Standards of Reporting Trials; HR: Heart rate; Pts: Patients; RR dia: Diastolic blood pressure; RR sys: Systolic blood pressure; SAA: Salivary alpha amylase; SD: Standard deviation; STAI: State-Trait Anxiety Inventory; VAS-A: Visual analogue scale for anxiety

\section{Acknowledgements}

We'd like to thank the dedicated team of midwives of the department of gynaecology and obstetrics of the Heinrich-Heine-University Düsseldorf.

\section{Funding}

The study was funded by a research grant from the Anton-Betz-Foundation.

\section{Availability of data and materials}

The dataset used and analysed during the current study is available from the corresponding author on reasonable request.

\section{Authors' contributions}

PH and NKS managed the project. PH, CH, WG, WJ and NKS conceived and designed the study. CH, JG, PB lead the acquisition of data. PH, JG and NKS analysed the data. PH, OTW, WG, WJ, MF, TF, NKS interpreted the data. JG, $\mathrm{PB}$, and MF provided administrative, technical and logistic support. PH and NKS drafted the manuscript. All authors revised the manuscript critically for important intellectual content and approved the final version of the manuscript.

\section{Ethics approval and consent to participate}

The study protocol was approved by the ethics committee of the Medical Department of the Heinrich-Heine-University in Dusseldorf (No.: 3625). The research was conducted in accordance with the 1964 Helsinki Declaration. All patients gave their written consent.

\section{Consent for publication}

Not applicable.

\section{Competing interests}

All authors declare that they have no competing interests.

\section{Publisher's Note}

Springer Nature remains neutral with regard to jurisdictional claims in published maps and institutional affiliations.

\footnotetext{
Author details

${ }^{1}$ Clinic for Gynecology and Obstetrics, Helios University Hospital Wuppertal, University Witten/Herdecke, Heusnerstr 40, 42283 Wuppertal, Germany. ${ }^{2}$ Clinic for Gynecology and Obstetrics, Heinrich-Heine-University, Düsseldorf, Germany. ${ }^{3}$ Department of Cognitive Psychology, Institute of Cognitive Neuroscience, Faculty of Psychology, Ruhr-University Bochum, Bochum, Germany. ${ }^{4}$ Musikerambulanz, Heinrich-Heine-University, Düsseldorf, Germany. ${ }^{5}$ Clinic for Gynecology and Obstetrics, University Hospital Ulm, Ulm, Germany. ${ }^{6}$ Department of Experimental Psychology, Heinrich-Heine-University, Düsseldorf, Germany.
}

Received: 1 August 2018 Accepted: 19 October 2018

Published online: 03 November 2018

\section{References}

1. Blüml V, Stammler-Safar M, Reitinger AK, Resch I, Naderer A, Leithner K. A qualitative approach to examine women's experience of planned cesarean. J Obstet Gynecol Neonatal Nurs. 2012;41:E82-90. https://doi.org/10.1111/j. 1552-6909.2012.01398.x.

2. Hepp P, Hagenbeck C, Burghardt B, Jaeger B, Wolf OT, Fehm T, et al. Measuring the course of anxiety in women giving birth by caesarean section: a prospective study. BMC Pregnancy Childbirth. 2016;16:113. https://doi.org/10.1186/s12884-016-0906-z.

3. Mulder EJH, Robles de Medina PG, Huizink AC, den Bergh BRH V, Buitelaar $J K$, GHA V. Prenatal maternal stress: effects on pregnancy and the (unborn) child. Early Hum Dev. 2002;70:3-14 http://www.ncbi.nlm.nih.gov/pubmed/ 12441200. Accessed 15 Oct 2017

4. O'Donnell KJ, Glover V, Barker ED, O'Connor TG. The persisting effect of maternal mood in pregnancy on childhood psychopathology. Dev Psychopathol. 2014;26:393-403. https://doi.org/10.1017/S0954579414000029.

5. Good M. Effects of relaxation and music on postoperative pain: a review. J Adv Nurs. 1996;24:905-14 http://www.ncbi.nlm.nih.gov/pubmed/8933249. Accessed 15 Oct 2017.

6. Powell R, Scott NW, Manyande A, Bruce J, Vögele C, Byrne-Davis LM, et al. Psychological preparation and postoperative outcomes for adults undergoing surgery under general anaesthesia. In: Powell R, editor. Cochrane Database of Systematic Reviews. Chichester: John Wiley \& Sons, Ltd; 2016. p. CD008646. https://doi.org/10.1002/14651858.CD008646.pub2.

7. Dewey KG. Maternal and fetal stress are associated with impaired lactogenesis in humans. J Nutr. 2001;131:3012S-5S http://www.ncbi.nlm.nih. gov/pubmed/11694638. Accessed 15 Oct 2017.

8. Grajeda R, Pérez-Escamilla R. Stress during labor and delivery is associated with delayed onset of lactation among urban Guatemalan women. J Nutr. 2002;132:3055-60 http://www.ncbi.nlm.nih.gov/pubmed/12368395. Accessed 15 Oct 2017

9. Conrad C. Music for healing: from magic to medicine. Lancet (London, England). 2010;376:1980-1 http://www.ncbi.nlm.nih.gov/pubmed/21171230. Accessed 15 Oct 2017

10. Goertz W, Dominick K, Heussen N, Vom dahl J. Music in the cath lab: who should select it. Clin Res Cardiol. 2011;100:395-402. https://doi.org/10.1007/ s00392-010-0256-1.

11. Smolen D, Topp R, Singer $L$. The effect of self-selected music during colonoscopy on anxiety, heart rate, and blood pressure. Appl Nurs Res. 2002;15:126-36 http://www.ncbi.nlm.nih.gov/pubmed/12173164. Accessed 14 Oct 2015

12. Koelsch S, Fuermetz J, Sack U, Bauer K, Hohenadel M, Wiegel M, et al. Effects of music listening on cortisol levels and Propofol consumption during spinal anesthesia. Front Psychol. 2011;2:58. https://doi.org/10.3389/fpsyg. 2011.00058.

13. Angioli R, De Cicco NC, Plotti F, Cafà EV, Dugo N, Damiani P, et al. Use of music to reduce anxiety during office hysteroscopy: prospective randomized trial. J Minim Invasive Gynecol. 2014;21:454-9. https://doi.org/ 10.1016/j.jmig.2013.07.020.

14. Hole J, Hirsch M, Ball E, Meads C. Music as an aid for postoperative recovery in adults: a systematic review and meta-analysis. Lancet. 2015;386:1659-71. https://doi.org/10.1016/S0140-6736(15)60169-6.

15. Laopaiboon M, Lumbiganon P, Martis R, Vatanasapt P, Somjaivong B. Music during caesarean section under regional anaesthesia for improving maternal and infant outcomes. Cochrane Database Syst Rev. 2009: CD006914. https://doi.org/10.1002/14651858.CD006914.pub2.

16. Li Y, Dong Y. Preoperative music intervention for patients undergoing cesarean delivery. Int J Gynaecol Obstet. 2012;119:81-3. https://doi.org/10, 1016/j.jigo.2012.05.017

17. Kushnir J, Friedman A, Ehrenfeld M, Kushnir T. Coping with preoperative anxiety in cesarean section: physiological, cognitive, and emotional effects of listening to favorite music. Birth. 2012;39:121-7. https://doi.org/10.1111/j. 1523-536X.2012.00532.x.

18. Chang S, Chen C. Effects of music therapy on women's physiologic measures, anxiety, and satisfaction during cesarean delivery. Res Nurs Health. 2005;28:453-61. https://doi.org/10.1002/nur.20102.

19. Reza N, Ali SM, Saeed K, Abul-Qasim A, Reza TH. The impact of music on postoperative pain and anxiety following cesarean section. Middle East J 
Anaesthesiol. 2007;19:573-86 http://www.ncbi.nlm.nih.gov/pubmed/ 18044285. Accessed 15 Oct 2017.

20. Laux L, Glanzmann P, Schaffner P, Spielberger CD. Das State-TraitAngstinventar (STAl) (Beltz-Test). Beltz Test GmbH: Göttingen; 1981.

21. Strahler J, Skoluda N, Kappert MB, Nater UM. Simultaneous measurement of salivary cortisol and alpha-amylase: application and recommendations. Neurosci Biobehav Rev. 2017;83:657-77. https://doi.org/10.1016/j.neubiorev. 2017.08.015.

22. Schoofs $\mathrm{D}$, Wolf OT. Are salivary gonadal steroid concentrations influenced by acute psychosocial stress? A study using the Trier social stress test (TSST). Int J Psychophysiol. 2011;80:36-43. https://doi.org/10.1016/j.jpsycho.2011.01.008.

23. Nilsson $U$. The anxiety- and pain-reducing effects of music interventions: a systematic review. AORN J. 2008;87:780-807. https://doi.org/10.1016/j.aorn. 2007.09.013

24. Petrakova L, Doering BK, Vits S, Engler H, Rief W, Schedlowski M, et al. Psychosocial stress increases salivary alpha-amylase activity independently from plasma noradrenaline levels. PLoS One. 2015;10:e0134561. https://doi. org/10.1371/journal.pone.0134561.

25. Faul F, Erdfelder E, Buchner A, Lang A-G. Statistical power analyses using $G^{*}$ power 3.1: tests for correlation and regression analyses. Behav Res Methods. 2009:41:1149-60. https://doi.org/10.3758/BRM.41.4.1149.

26. Ikonomidou E, Rehnström A, Naesh O. Effect of music on vital signs and postoperative pain. AORN J. 2004;80:269-74, 277-8. http://www.ncbi.nlm. nih.gov/pubmed/15382598 Accessed 15 Oct 2017.

27. Khalfa S, Bella SD, Roy M, Peretz I, Lupien SJ. Effects of relaxing music on salivary cortisol level after psychological stress. Ann N Y Acad Sci. 2003;999: 374-6 http://www.ncbi.nlm.nih.gov/pubmed/14681158. Accessed 15 Oct 2017.

28. Zengin S, Kabul S, Al B, Sarcan E, Doğan M, Yildirim C. Effects of music therapy on pain and anxiety in patients undergoing port catheter placement procedure. Complement Ther Med. 2013;21:689-96. https://doi.org/10.1016/j.ctim.2013.08.017.

29. van Stegeren AH, Wolf OT, Kindt M. Salivary alpha amylase and cortisol responses to different stress tasks: impact of sex. Int J Psychophysiol. 2008;69:33-40

30. Orbach-Zinger S, Ginosar Y, Elliston J, Fadon C, Abu-Lil M, Raz A, et al. Influence of preoperative anxiety on hypotension after spinal anaesthesia in women undergoing caesarean delivery. Br J Anaesth. 2012;109:943-9. https://doi.org/10.1093/bja/aes313.

31. Sadi H, Finkelman M, Rosenberg M. Salivary cortisol, salivary alpha amylase, and the dental anxiety scale. Anesth Prog. 2013;60:46-53. https://doi.org/10. 2344/0003-3006-60.2.46.

32. Koelsch S, Jäncke L. Music and the heart. Eur Heart J. 2015;36:3043-9. https://doi.org/10.1093/eurhearti/ehv430.

33. Koelsch S. Brain correlates of music-evoked emotions. Nat Rev Neurosci. 2014;15:170-80. https://doi.org/10.1038/nrn3666.

34. Bernardi L, Porta C, Casucci G, Balsamo R, Bernardi NF, Fogari R, et al. Dynamic interactions between musical, cardiovascular, and cerebral rhythms in humans. Circulation. 2009;119:3171-80 http://www.ncbi.nlm.nih.gov/ pubmed/19569263. Accessed 22 Oct 2017.

35. Bernatzky G, Kreutz G. Musik und Medizin Chancen für Therapie, Prävention und Bildung. Vienna: Springer; 2015.

36. Thaut MH, McIntosh GC, Hoemberg V. Neurobiological foundations of neurologic music therapy: rhythmic entrainment and the motor system. Front Psychol. 2014;5:1185. https://doi.org/10.3389/fpsyg.2014.01185.

37. Weldon S-M, Korkiakangas T, Bezemer J, Kneebone R. Music and communication in the operating theatre. J Adv Nurs. 2015;71:2763-74. https://doi.org/10.1111/jan.12744.

Ready to submit your research? Choose BMC and benefit from:

- fast, convenient online submission

- thorough peer review by experienced researchers in your field

- rapid publication on acceptance

- support for research data, including large and complex data types

- gold Open Access which fosters wider collaboration and increased citations

- maximum visibility for your research: over $100 \mathrm{M}$ website views per year

At BMC, research is always in progress.

Learn more biomedcentral.com/submissions 